\title{
Revisiting the Factors Underlying Maxillary Midline Diastema
}

\author{
Abdullah M. Zakria Jaija, ${ }^{1}$ Amr Ragab El-Beialy, ${ }^{2,3}$ and Yehya A. Mostafa ${ }^{2,3}$ \\ ${ }^{1}$ Private Practice, P.O. Box 376385, Riyadh 11335, Saudi Arabia \\ ${ }^{2}$ Department of Orthodontics and Dentofacial Orthopedics, Faculty of Oral and Dental Medicine, Cairo University, \\ 11 El-Saraya Street, Manial, Cairo 12511, Egypt \\ ${ }^{3}$ MOrth Programs at Cairo University and Future University, Cairo, Egypt
}

Correspondence should be addressed to Amr Ragab El-Beialy; amr.elbeialy@dentistry.cu.edu.eg

Received 5 December 2015; Accepted 17 March 2016

Academic Editor: William Maloney

Copyright (c) 2016 Abdullah M. Zakria Jaija et al. This is an open access article distributed under the Creative Commons Attribution License, which permits unrestricted use, distribution, and reproduction in any medium, provided the original work is properly cited.

Aim. The aim of this study is to analyze the etiological factors underlying the presence of maxillary midline diastema in a sample of orthodontic patients. Materials and Methods. One hundred patients who fulfill the inclusion criteria were selected from 1355 patients seeking orthodontic treatment. The pretreatment orthodontic records were analyzed. The width of the maxillary midline diastema was measured clinically with a digital caliper at two levels: the mesioincisal angles of the central incisors and five millimeters from the incisal edge. The two measurements were averaged, and patients with diastema of more than 0.5 millimeter in width were enrolled. Results. Diastema is a multifactorial clinical finding with more than one underlying etiological cause. The interrelationship between the familial pattern of midline diastema and the microdontia, macroglossia, labial frenum, and alveolar cleft conforms was clear. The effect of a mesiodens and the upper lateral incisor whether bilaterally missing, unerupted, or peg shaped was minimal. Conclusion. Etiological factors underlying maxillary midline diastema are interconnected. Using a checklist as a guide during handling maxillary midline diastema is important in the different stages of treatment.

\section{Introduction}

The presence of a midline diastema represents an esthetic and psychological impairment and distress for patients seeking orthodontic treatment [1]. Maxillary midline diastema is a clinical sign, which has a multitude of underlying etiological factors that might be interdependent or independent.

Many etiological factors for maxillary midline diastema have been reported in the literature [2]. Among them are the physiological (developmental) self-limiting diastema, familial background, mesiodens [3-5], abnormal labial frenum [6-8], missing or undersized lateral incisor, thumb sucking, mouth breathing, tongue thrust, ankylosed central incisor, flared or rotated central incisors, anodontia, macroglossia $[9,10]$, dentoalveolar disproportion, generalized spacing [11], localized spacing, closed bite, facial type, ethnic and familial characteristics $[12,13]$, interpremaxillary suture and transseptal fibers [14], midline pathology, midline submucosal alveolar cleft [15], tongue piercing [16], gingival recession, and pathological tooth migration [17].
The aim of this survey is to investigate the correlation between the different etiological factors underlying maxillary midline diastema and highlight their clinical implications.

\section{Materials and Methods}

This survey was performed on patients undergoing orthodontic treatment at three orthodontic graduate programs. The pretreatment records of 1355 patients were examined, to collect 100 patients fulfilling the inclusion criterion (presence of maxillary midline diastema $>0.5 \mathrm{~mm}$ ) within an age range of 13-30 years. The pretreatment records included history, intra- and extraoral examination, and panoramic and periapical radiographs of the maxillary incisor region. The width of the maxillary midline diastema was measured clinically with a digital caliper at two levels: the mesioincisal angles of the central incisors and five millimeters from the incisal edge. The two measurements were averaged, and patients with diastema of more than 0.5 millimeter in 


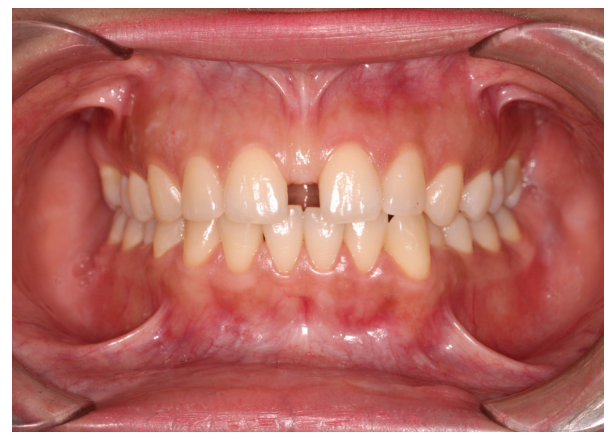

FIGURE 1: Maxillary midline diastema.

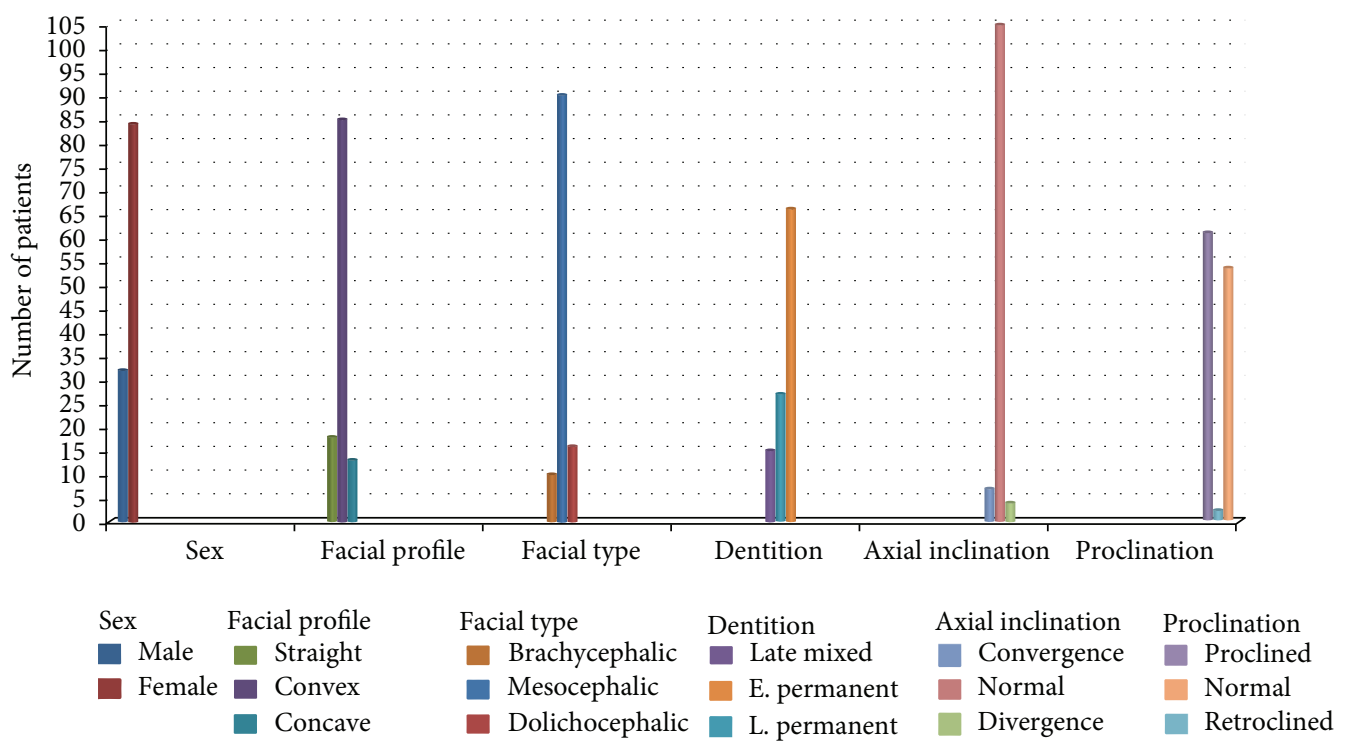

FIGURE 2: Bar chart showing the distribution of the criteria of the sample.

width were enrolled (Figure 1). The examination was made by the principle observer and repeated by the second observer.

Because of the physiological diastema, patients younger than 13 years were excluded, while patients above 30 years were excluded because of the possibility of diastema formation due to periodontal involvement and migration of teeth.

The distribution of the criteria of the sample was analyzed (Figure 2). The criteria represent the commonsensical orthodontic categories that segregate the sample into comparable subclasses. The etiological factors underlying the maxillary midline diastema were extracted from the records and clinical examination of the patients (Table 1). These etiological foundations were separated into major etiological factors and etiological factors of lesser influence. These factors represent all the etiological factors underlying the presence of the maxillary midline diastema that were extracted from the research sample. The prevalence of each factor in percentage of the 100 cases enrolled was calculated.

Additionally, the association between diastema with overjet and overbite is depicted through dividing the sample into 10 groups each representing $1 \mathrm{~mm}$ regarding diastema, overjet, and overbite (Figure 3).

\section{Results}

The prevalence of the diastema was found to be $13.6 \%$ among the screened sample. The occurrence of the six criteria (Figure 2) demonstrated that the maxillary midline diastema is more observed in females, mesocephalic faces, convex facial profiles, and the early permanent dentition. Maxillary midline diastema is more prevalent with upright maxillary central incisors than convergent or divergent central incisors. The least prevalence of diastema occurs with retroclined maxillary incisors.

The relationship between the maxillary midline diastema, overjet, and overbite depicted in Figure 3 shows that a diastema width of 1-2 mm is more prevalent (44 patients) than other extents of diastema, and this prevalence decreases as the amount of overbite and overjet increases.

Etiological factors were segregated into major contributing factors and factors of lesser contribution taking 5\% prevalence as the limit (Table 1). The interrelation (overlap) between the major contributing factors is denoted by intersecting circles charts (Figures 4-6). Factors that might be of strong developmental interrelation were linked together in 


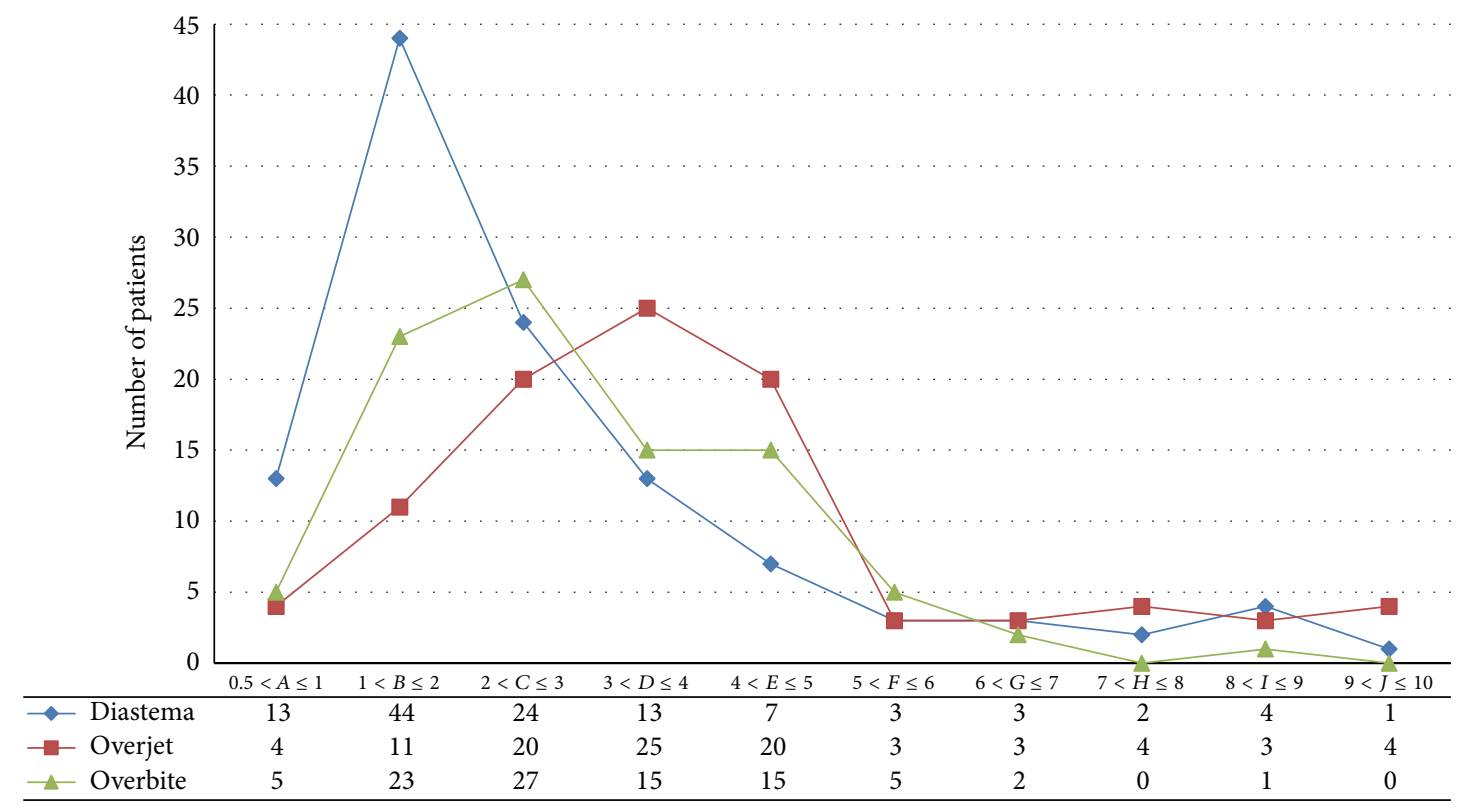

FIGURE 3: Chart showing the relation of common prevalence between diastema, overjet, and overbite.

TABLE 1: The etiological factors of the maxillary midline diastema extracted from the sample.

\begin{tabular}{lccc}
\hline Major etiological factors & \% of prevalence & Etiological factors of lesser prevalence & $\%$ of prevalence \\
\hline Generalized spacing & 42 & Missing bilateral maxillary central incisors & 5 \\
Familial incidence & 39 & Bilateral missing maxillary lateral incisors & Peg shaped maxillary lateral incisors \\
Abnormal frenal attachment & 22 & Missing unilateral maxillary central incisors & 5 \\
Alveolar intraosseous cleft & 21 & Ankylosed maxillary central incisors & 4 \\
Tongue-thrusting & 16 & Excess bony defect & Thumb sucking \\
Macroglossia & 15 & Mesiodens & 1 \\
Unerupted canine bilateral & 14 & Malformed maxillary central incisors \\
Unerupted canine (unilateral) & 12 & Genetics \\
Microdontia & 10 & Midline pathosis \\
Unilateral missing maxillary lateral incisors & 8 & Unerupted maxillary lateral incisors \\
Palatally erupted maxillary lateral incisors & 6 & 0 \\
Mouth breathing & 6 & 0 \\
Tooth migration & 6 & 0
\end{tabular}

a single chart. The areas of intersection represent the number of cases where more than one major contributing factor exists.

\section{Discussion}

Treatment of maxillary midline diastema should be directed towards management of the underlying cause before seeking closure of the diastema; thus, identifying the etiology is of chief importance. The aim of this survey is to highlight the factors underlying maxillary midline diastema and the interrelation between them. This might influence the timing for closure of the diastema during treatment and/or retention protocols.
Our results conformed to the consensus that diastema is a multifactorial clinical finding with more than one underlying etiological cause. Based on a prevalence of $5 \%$, the etiological factors were segregated into major and minor factors. Surprisingly, the effect of the upper lateral incisor whether bilaterally missing, unerupted, or peg shaped was minimal. The same outcome was found with a mesiodens. The interrelationship between the familial pattern of midline diastema and the microdontia, macroglossia, labial frenum, and alveolar cleft conforms was clear. On the other hand, no cases showed a familial tendency of missing unilateral maxillary lateral incisor. However, as regards the enlarged labial frenum as an etiological cause, results of this study revealed that it represents only a minor etiological cause, 
TABLE 2: Checklist showing the impact of each etiological factor of the maxillary midline diastema upon the diagnosis, treatment, or retention protocol.

\begin{tabular}{|c|c|c|c|}
\hline Factor & Extra diagnostic tool & Treatment modification & Retention modification \\
\hline Generalized spacing & & & $\square$ (Permanent) \\
\hline Familial incidence & $\square$ (Family screening) & & $\square$ (Permanent) \\
\hline Abnormal frenal attachment & $\square$ (Periapical radiograph) & $\square$ (Frenotomy) & $\square$ (Prolonged) \\
\hline Alveolar intraosseous cleft & $\square$ (Periapical radiograph) & $\square$ (Nonidentified) & \\
\hline Tongue-thrusting & & $\square$ (Habit breaking appliance) & $\square$ (Habit breaking) \\
\hline Macroglossia & & $\square$ (No encroaching on tongue) & $\square$ (Permanent) \\
\hline \multicolumn{4}{|l|}{ Unerupted canine bilaterally } \\
\hline \multicolumn{4}{|l|}{ Unerupted canine unilaterally } \\
\hline Microdontia & & $\square$ (Build-up) & \\
\hline Unilateral missing maxillary lateral incisors & & $\square$ (Prosthesis) $\square$ (Canine substitution) & \\
\hline Palatally erupted maxillary lateral incisors & & $\square$ (Root torquing) & \\
\hline Mouth breathing & $\square$ (ENT consultation) & & $\square$ (Habit breaking) \\
\hline \multicolumn{4}{|l|}{ Tooth migration } \\
\hline Missing bilateral maxillary central incisors & & $\square$ (Prosthesis) & \\
\hline Bilateral missing maxillary lateral incisors & & $\square$ (Prosthesis) $\square$ (Canine substitution) & \\
\hline Peg shape maxillary lateral incisors & & $\square$ (Build-up) & \\
\hline Missing unilateral maxillary central incisors & & $\square$ (Prosthesis) & \\
\hline Ankylosed maxillary central incisors & & $\square$ (Luxation, crowning, extraction) & \\
\hline Excess bony defect & & $\square$ (Surgical) & \\
\hline Thumb sucking & & $\square$ (Habit breaking appliance) & \\
\hline Mesiodens & & $\square$ (Surgical extraction) & \\
\hline Malformed maxillary central incisors & & $\square$ (Build-up) & \\
\hline Midline pathosis & $\square$ (Periapical radiograph) & $\square$ (Surgical excision) & \\
\hline Unerupted maxillary lateral incisors & $\square$ (Periapical radiograph) & & \\
\hline
\end{tabular}

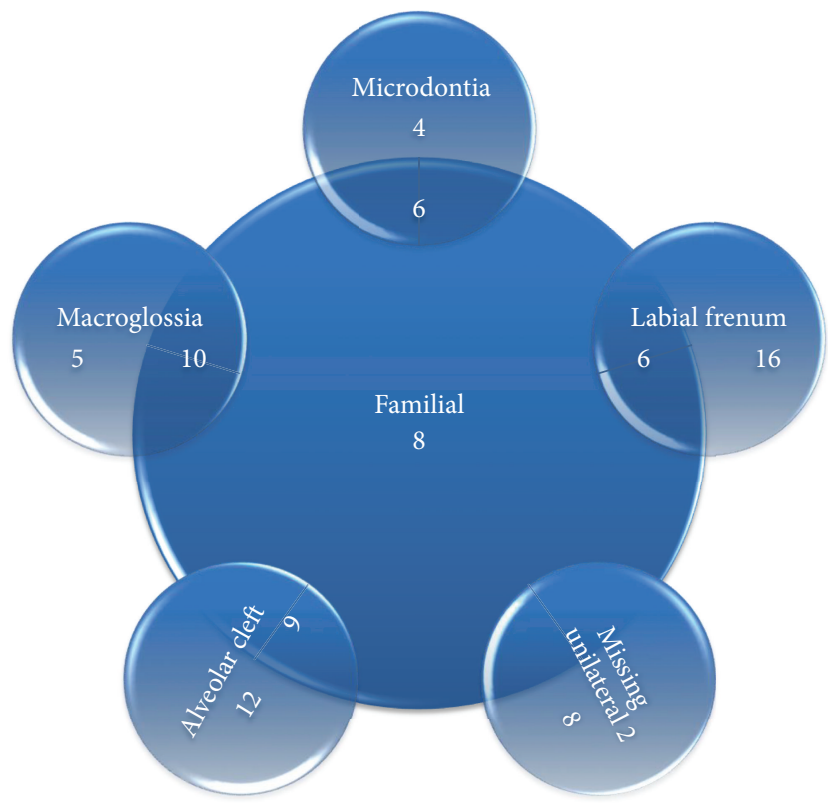

FIGURE 4: Intersecting circles chart depicting the common occurrence between the major contributing factors.

an observation that conforms to the findings of Huang and Creath [2]. In addition, the interrelation between the alveolar cleft and abnormal labial frenum was an important finding.

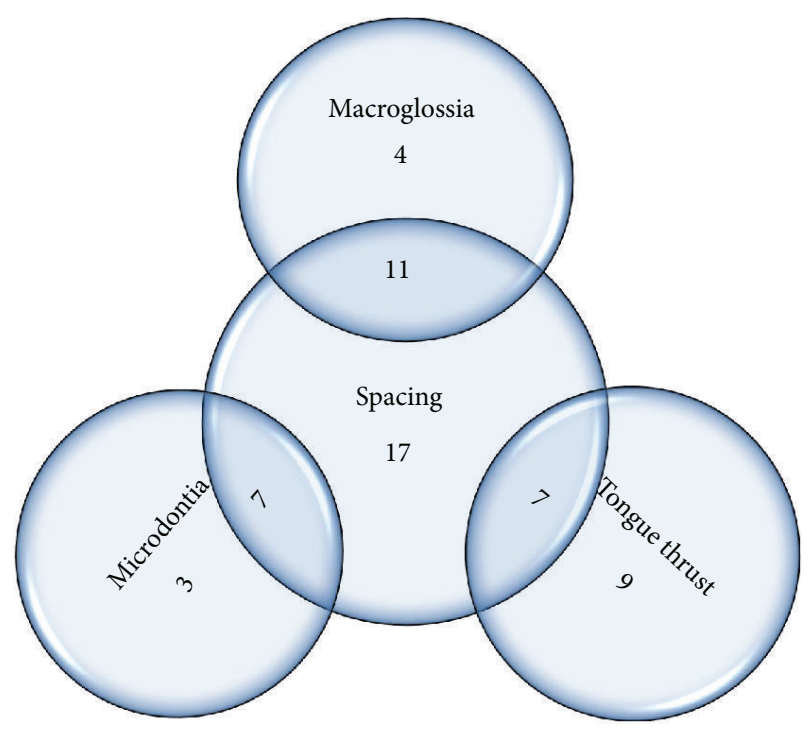

FIGURE 5: Intersecting circles chart depicting the common occurrence between spacing, microdontia, macroglossia, and tongue thrust.

Implementation of the findings of this survey is important from the clinical sense. The impact of each etiological factor of the maxillary midline diastema upon the diagnosis, treatment, or retention protocol is summarized into a checklist. This checklist was designed to highlight the intervention at 


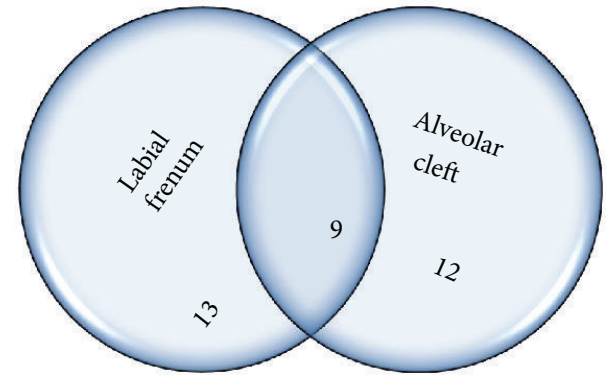

FIGURE 6: Intersecting circles chart depicting the common occurrence between labial frenum and alveolar cleft.

the different stages of treatment for each etiological factor (Table 2).

\section{Conclusion}

Etiological factors underlying maxillary midline diastema are interconnected.

Using a checklist as a guide during handling maxillary midline diastema is important in the different stages of treatment.

\section{Competing Interests}

The authors declare that they have no competing interests.

\section{References}

[1] N. Gkantidis, O.-E. Kolokitha, and N. Topouzelis, "Management of maxillary midline diastema with emphasis on etiology," Journal of Clinical Pediatric Dentistry, vol. 32, no. 4, pp. 265-272, 2008.

[2] W. J. Huang and C. J. Creath, "The midline diastema: a review of its etiology and treatment," Pediatric Dentistry, vol. 17, no. 3 , pp. 171-179, 1995.

[3] N. B. Nagaveni, K. V. Umashankara, Sreedevi, B. P. Reddy, N. B. Radhika, and T. S. Satisha, "Multi-lobed mesiodens with a palatal talon cusp: a rare case report," Brazilian Dental Journal, vol. 21, no. 4, pp. 375-378, 2010.

[4] K. Gündüz, P. Celenk, Z. Zengin, and P. Sümer, "Mesiodens: a radiographic study in children," Journal of Oral Science, vol. 50, no. 3, pp. 287-291, 2008.

[5] A. Alaçam and M. Bani, "Mesiodens as a risk factor in treatment of trauma cases," Dental Traumatology, vol. 25, no. 2, pp. e25e31, 2009.

[6] D. Belic and K. Obrez-Oblak, "Diastema verum and persistent labial frenum," Zobozdravstveni Vestnik, vol. 45, no. 4-5, pp.107109, 1990.

[7] J. G. Edwards, "The diastema, the frenum, the frenectomy: a clinical study," American Journal of Orthodontics, vol. 71, no. 5, pp. $489-508,1977$.

[8] S. Bagga, K. M. Bhat, G. S. Bhat, and B. S. Thomas, "Esthetic management of the upper labial frenum: a novel frenectomy technique," Quintessence International, vol. 37, no. 10, pp. 819$823,2006$.

[9] N. Topouzelis, C. Iliopoulos, and O. E. Kolokitha, "Macroglossia," International Dental Journal, vol. 61, no. 2, pp. 63-69, 2011.
[10] G. Farronato, S. Salvadori, L. Giannini, and C. Maspero, "Congenital macroglossia: surgical and orthodontic management," Progress in Orthodontics, vol. 13, no. 1, pp. 92-98, 2012.

[11] F. Popovich and G. W. Thompson, "Maxillary diastema: indications for treatment," American Journal of Orthodontics, vol. 75, no. 4, pp. 399-404, 1979.

[12] J. R. Gass, M. Valiathan, H. K. Tiwari, M. G. Hans, and R. C. Elston, "Familial correlations and heritability of maxillary midline diastema," American Journal of Orthodontics and Dentofacial Orthopedics, vol. 123, no. 1, pp. 35-39, 2003.

[13] M. Yamaoka, K. Furusawa, M. Tanaka, and H. Tanaka, "Unerupted canine without median diastema," Journal of Oral Rehabilitation, vol. 24, no. 6, pp. 454-456, 1997.

[14] R. Stubley, "The influence of transseptal fibers on incisor position and diastema formation," American Journal of Orthodontics, vol. 70, no. 6, pp. 645-662, 1976.

[15] H.-T. Liao, C.-H. Chen, L. Bergeron, E. W.-C. Ko, P. K. T. Chen, and Y.-R. Chen, "Alveolar bone grafting in the treatment of midline alveolar cleft and diastema in incomplete median cleft lip," International Journal of Oral and Maxillofacial Surgery, vol. 37, no. 10, pp. 886-891, 2008.

[16] S. Tabbaa, I. Guigova, and C. B. Preston, "Midline diastema caused by tongue piercing," Journal of Clinical Orthodontics, vol. 44, no. 7, pp. 426-428, 2010.

[17] A. R. Pradeep and D. C. G. Sharma, "Gingival recession and pathologic migration due to an unusual habit," Journal of the International Academy of Periodontology, vol. 8, no. 3, pp. 7477, 2006. 


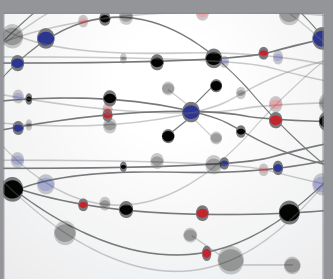

The Scientific World Journal
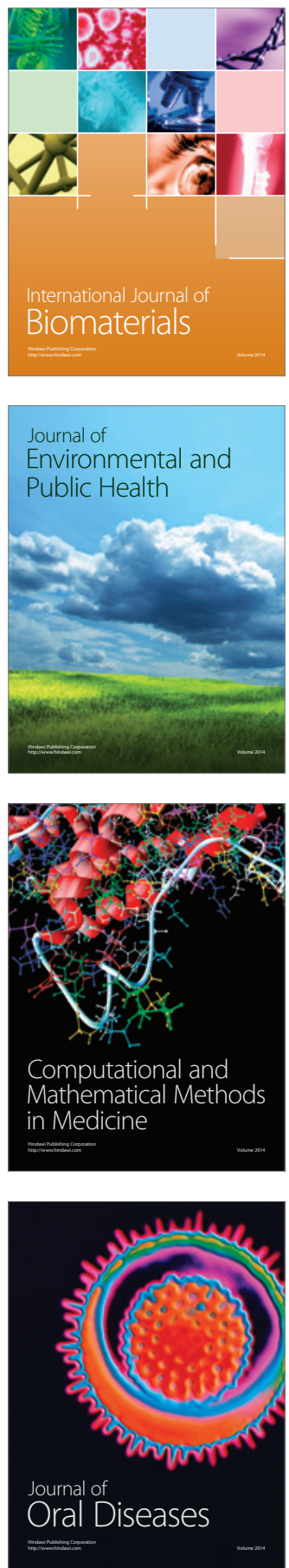
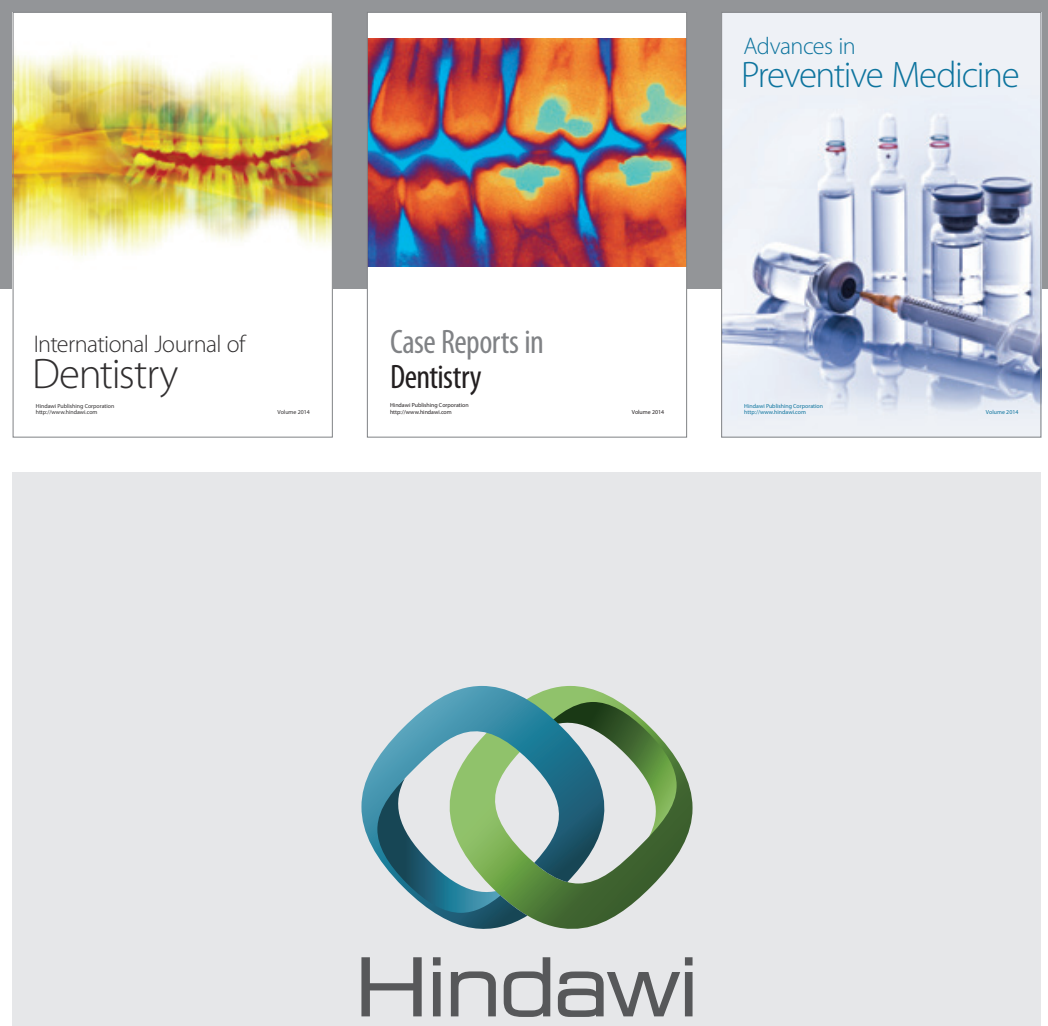

Submit your manuscripts at

http://www.hindawi.com
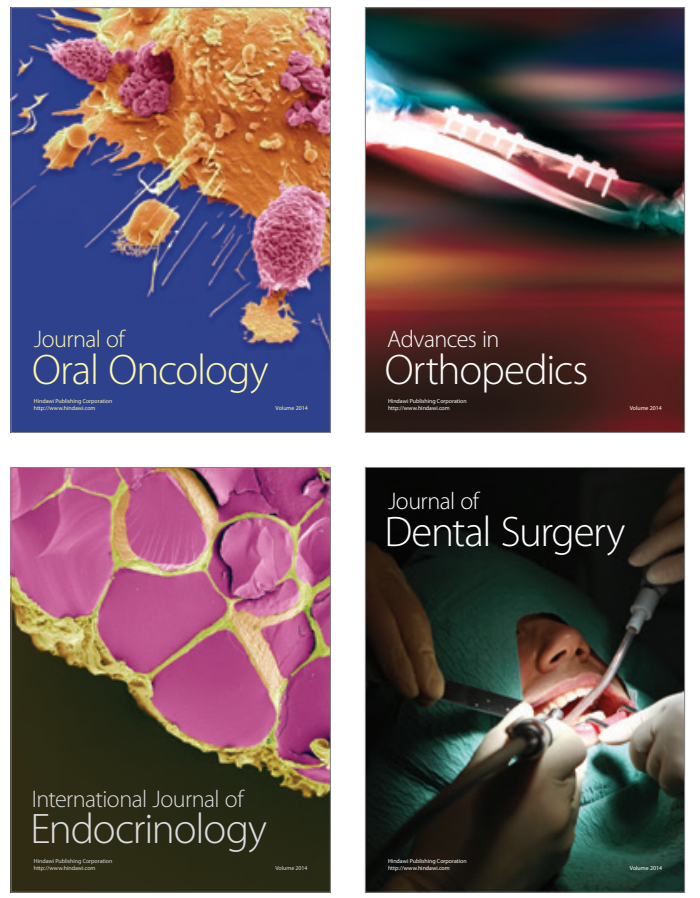
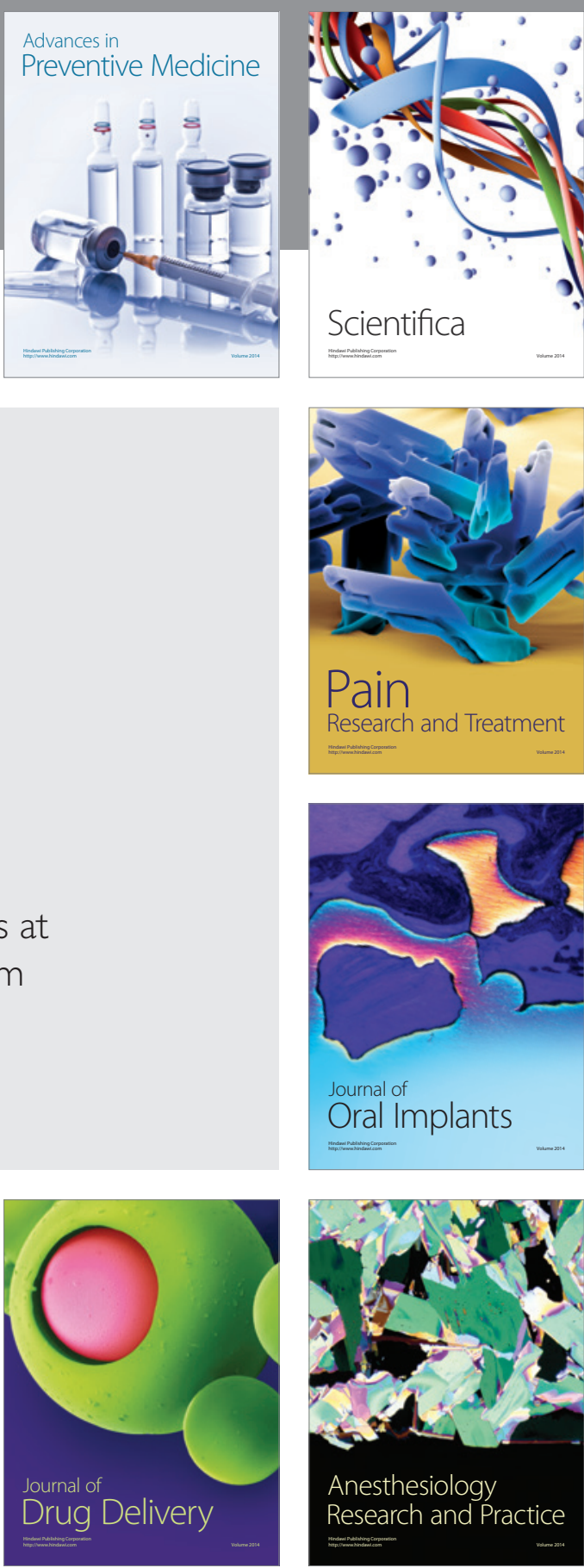

Scientifica
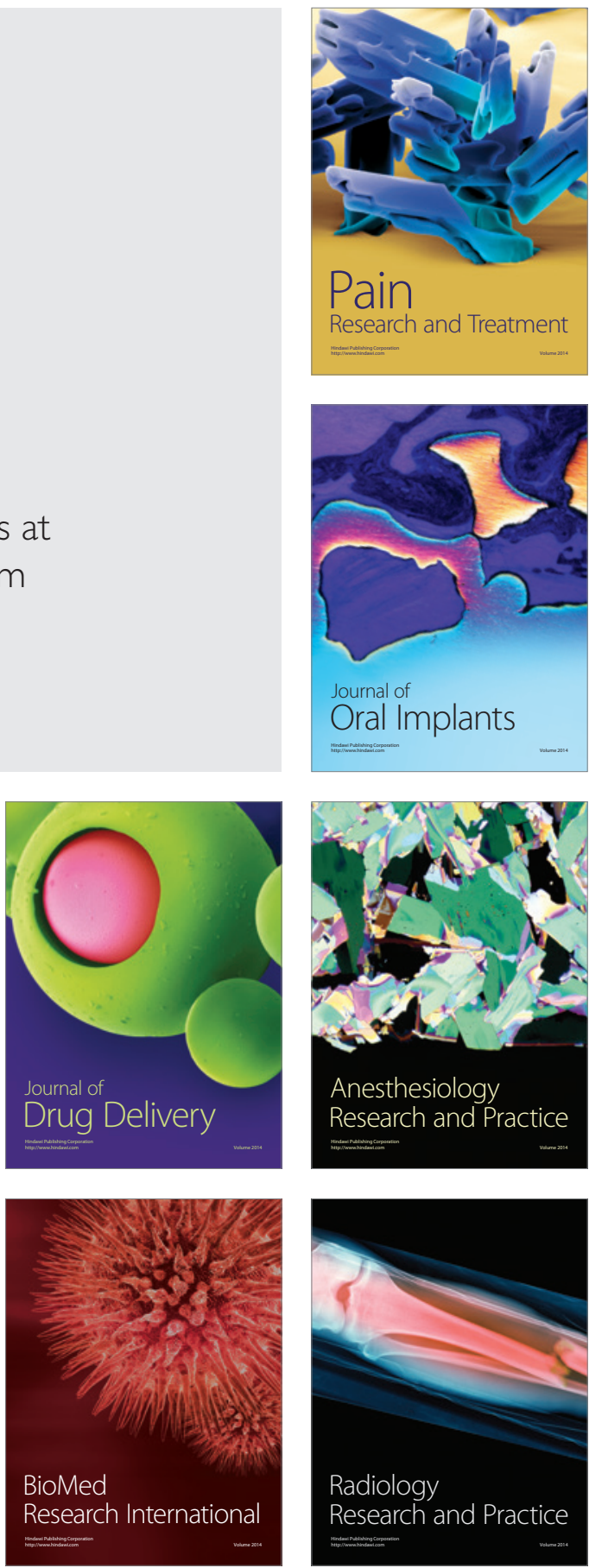